\title{
Correlation Function in Ising Models
}

\author{
C. Ruge ${ }^{a}$, P. Zhu ${ }^{b}$ and F. Wagner ${ }^{a}$
}

a) Institut für Theoretische Physik und Sternwarte

Univ. Kiel, D-24098 Kiel, Germany

E Mail: ruge@theo-physik.uni-kiel.de

b) Dept. of Phys. Hangzou Univ.

Hangzou, 310028, P.R. China

\begin{abstract}
We simulated the fourier transform of the correlation function of the Ising model in two and three dimensions using a single cluster algorithm with improved estimators. The simulations are in agreement with series expansion and the available exact results in $d=2$, which shows, that the cluster algorithm can succesfully be applied for correlations. We show as a further result that our data do not support a hypothesis of Fisher that in any $d=2$ lattice the fourier transform of the correlation function depends on the lattice generating function only. In $d=3$ our simulation are again in agreement with the results from the series expansion, except for the amplitudes $f_{ \pm}$, where we find $f_{+} / f_{-}=2.06(1)$.
\end{abstract}




\section{Introduction}

A simple way to characterize the behaviour of spin variables of an Ising model consists in the two point correlation function $g_{x, y}$. Except for the trivial $d=1$ case a general expression has not been found. In $d=2$ are only the exact expressions for small and large separations known [1]. Apart from that most information comes from high temperature expansions [2, 3, 4]. Universal quantities related to the critical behaviour with scaling dimension 0 can be calculated from $\phi^{4}$ field theory [5, 6, 7]. Numerical simulations are difficult for several reasons. Direct simulation of $g_{x, y}$ requires an computational effort increasing with $N^{2}$, where $N$ is the number of lattice points. Within periodic boundary conditions $g$ is translational invariant and can be reduced to the fourier transform of $g_{0, x}$. Since this still needs an effort increasing with $N$, only lattices of rather modest linear extension can be treated for $d>1$. For many applications the knowledge of the fourier transform along one or two directions is sufficient which renders us a feasible problem. Even within that restriction success depends on the algorithm. In general one may adopt a single spin update (Metropolis or Heatbath algorithm [8]) or a cluster

algorithm [9, 10]. For thermal quantities the smaller autocorrelation time of the latter is compensated by the better vectorization of the former algorithm [11]. For the fourier transform of the correlation function one has to measure $\left|\sum_{x} e^{i k x} \sigma_{x}\right|^{2}$. Since both the spin variable $\sigma_{x}$ and the phase factor vary rapidly with $x$, the resulting fluctuations prevent any accurate measurement. The problem of fluctuation is particular striking approaching the critical temperature. The improved estimators in a cluster algorithm have much less variation and lead to more accurate results. The aim of the present paper is to show that the correlation function can be reliably determined with the single cluster algorithm of Wolff [10] by comparing our results with known exact results in $d=2$ and the results from series expansion in $d=2$ and $d=3$ [2, 3, 12].

The paper is organized in the following way. In Section 2 we collect the necessary formalism on the correlation function. The cluster algorithm is described in section 3 and section 1 contains the results of our simulations. In section 5 we give our conclusions. 


\section{Correlation functions}

The connected pair correlation function $g_{x, y}^{c}$ for an Ising model is defined as

$$
g_{x, y}^{c}=\left\langle\sigma_{x} \sigma_{y}\right\rangle-m^{2} \Theta\left(T_{c}-T\right)
$$

$x, y$ denote the sites of a d-dimensional simple cubic lattice of linear dimension $L$ with periodic boundary conditions and $\langle A\rangle$ means the thermal average at temperature $T$ taken with an energy $E$

$$
E=\frac{1}{2} \sum_{x, y} \delta_{|x-y|, 1} \sigma_{x} \sigma_{y}
$$

where $\delta_{|x|, 1}$ projects on next neighbours of $x=0$.

Below the critical temperature a magnetization term has to be subtracted from $\left\langle\sigma_{x} \sigma_{y}\right\rangle$ in order to obtain a reasonable behaviour at large distances $|x-y| . g_{x, y}^{c}$ can be considered as a $L^{d} \cdot L^{d}$ matrix. Usually its inverse (the proper vertex function in field theory) has a simpler behaviour. By means of translation invariance we can write

$$
\left(g^{c}\right)_{x, y}^{-1}=\frac{1}{L^{d}} \sum_{k} e^{i k(x-y)} \frac{1}{\hat{g}(k)}
$$

$k$ is a $d$ dimensional vector whose components $k_{i}$ are restricted to the first Billouin zone $\left|k_{i}\right| \leq \pi$ and have the form $\frac{2 \pi}{L} n_{i}$ with integer $n_{i}$. The positive function $\hat{g}(k)$ is simply the fourier transform of $g_{0, x}^{c}$. For $|k|<<1$ it has the expansion

$$
\frac{1}{\hat{g}(k)}=\frac{1}{\chi(T)}\left(1+\xi^{2} k^{2}+\ldots\right)
$$

where $\chi(T)$ denotes the susceptibility in units of the Curie susceptibility and $\xi(T)$ the effective correlation length. Near $T \sim T_{c}$ we expect the following dependance on the scaling variable $\tau=1-\frac{T}{T_{c}}$ :

$$
\begin{aligned}
\chi(T) & =C_{ \pm}|\tau|^{-\gamma} \\
\xi(T) & =f_{ \pm}|\tau|^{-\nu} \\
m & =B \tau^{\beta} .
\end{aligned}
$$

The \pm signs in the amplitudes refer to $T \stackrel{\gtrless}{<} T_{c}$. In $d=2$ the critical indices and the amplitudes $C$ and $B$ are known exactly [13, 14, 15, 16, 17, but the 
exact known $f_{ \pm}^{t}$ refer to the true correlation length $\xi^{t}$ which is defined by the exponential decay of the correlation function $\ln g_{x, y} \sim-|x-y| / \xi^{t}$ for large separations and may differ especially for $T<T_{c}$ from $\xi$. Theoretical information on $f_{ \pm}$in $d=2$ and $d=3$ comes from series expansion [2, 3] and on the ratio $f_{+} / f_{-}$in $d=3$ from renormalized perturbation theory [5]. As discussed in the next section $\hat{g}(k)$ can be estimated by numerical simulations only at special values of the arguments. In the following we adopt the choices $k_{R}=k_{0}(1,1, . .1)$ (radial direction) and $k_{L}=k_{0}(1,0, . .0)$ (linear direction). This corresponds to two functions $g_{L}\left(k_{0}\right)$ and $g_{R}\left(k_{0}\right)$ depending only on a scalar argument $k_{0}$ :

$$
\begin{aligned}
& g_{L}\left(k_{0}\right)=\hat{g}\left(k_{L}\right) \\
& g_{R}\left(k_{0}\right)=\hat{g}\left(k_{R}\right)
\end{aligned}
$$

Due to the rotationally invariant form of eq(4) $\chi$ and $\xi$ can be determined from one of the functions (6). In $d=3$ we will restrict our numerical analysis to the small $k$ region. In $d=2$ we can test in addition the following hypothesis stated by Fisher et. al. [2]. Motivated by the results of the high temperature expansion they conjectured, that for all $d=2$ Ising models $\hat{g}(k)$ is a function of the lattice generating function $q(k)$ only which is given on a square lattice by

$$
q(k)=2\left(\cos \left(k_{1}\right)+\cos \left(k_{2}\right)\right) .
$$

Since $\hat{g}(q(k))$ is a function of one variable only, $g_{L}$ and $g_{R}$ must satisfy the following relation

$$
g_{R}\left(k_{0}^{\prime}\right)=g_{L}\left(k_{0}\right)
$$

provided $k_{0}^{\prime}$ is connected to $k_{0}$ by

$$
\sin ^{2}\left(k_{0} / 2\right)=2 \sin ^{2}\left(k_{0}^{\prime} / 2\right) .
$$

Even if eq(8) would not be true, expansion of $1 / \hat{g}$ in powers of $q$ consists in a very accurate representation of $g_{x, y}^{c}$. Writing

$$
\frac{1}{\hat{g}(k)}=1-\sum_{n} H_{n}(T)(q(k))^{n}
$$

only $H_{n}$ with $n \leq 2$ are present up to $o(1 / T)^{12}$. Converting eq.(10) in position space we get from eq.(3)

$$
\begin{aligned}
\left(g^{c}\right)_{x, y}^{-1} & =a_{0}(T) \delta_{x, y}-a_{1}(T) \delta_{|x-y|, 1} \\
& -a_{2}(T)\left(\delta_{|x-y|, 2}+2 \delta_{|x-y|, \sqrt{2}}\right)+o(1 / T)^{12}
\end{aligned}
$$


where $\delta_{|x-y|, r}$ projects on points $x, y$ having euclidean distance $r$. The high temperature expansion of ref [2] for $a_{i}(T)$ are given for convenience in appendix A. $a_{2}(T)$ is of order $\left(\frac{1}{T}\right)^{10}$ and therefore very small. If the expansion (11) is valid, the coefficients $a_{i}$ can be determined from low order moments of the function (6)

$$
\begin{aligned}
& a_{0}(T)=\frac{1}{2 L} \sum_{k_{0}}\left[\frac{2+\cos k_{0}}{g_{R}\left(k_{0}\right)}+\frac{\cos k_{0}}{g_{L}\left(k_{0}\right)}\right] \\
& a_{1}(T)=\frac{1}{2 L} \sum_{k_{0}} \frac{\cos k_{0}}{g_{R}\left(k_{0}\right)} \\
& a_{2}(T)=\frac{1}{L} \sum_{k_{0}} \frac{\cos 2 k_{0}}{g_{L}\left(k_{0}\right)} .
\end{aligned}
$$

Note, that the sum $\sum_{k_{0}}$ is only one dimensional. For $1 / T<0.4$ the first two terms in eq.(11) give a very accurate representation of $\left(g^{c}\right)_{x, y}^{-1}$, which may be useful in applications 18 .

\section{$3 \quad$ Numerical methods}

A direct simulation of the disconnected correlation function

$$
g_{x, y}=\left\langle\sigma_{x} \sigma_{y}\right\rangle
$$

is prohibitive both from storage requirement and computational effort, even the latter can be reduced by Boolean operations (since $\sigma_{x}$ are two valued quantities) and multi spin update [19]. Taking translation invariance within periodic boundary conditions into account only $g_{0, y}$ is needed, or

$$
g_{0, y}=\frac{1}{L^{d}} \sum_{x^{\prime}}\left\langle\sigma_{x^{\prime}} \sigma_{y+x^{\prime}}\right\rangle
$$

Having cured the storage problem the computational problem remains that eq. (14) requires $O\left(L^{d}\right)$ algebraic operations per single spin update. For the observables discussed in section 2 not the full fourier transform of $g$ is needed but rather $g_{L}\left(k_{0}\right)$ or $g_{R}\left(k_{0}\right)$ with $k_{0} \neq 0$

$$
g_{R, L}\left(k_{0}\right)=\frac{1}{L^{d}}\left\langle\left|\sum_{x} e^{-i\left(x \cdot k_{R, L}\right)} \sigma_{x}\right|^{2}\right\rangle .
$$


The value at $k_{0}=0$ has to be determined by an extrapolation $k_{0} \rightarrow 0$. The difference of this extrapolated value and the actual value of the r.h.s. of eq.(15) gives $m^{2} L^{d}$. Since $k_{0}$ in eq.(15) can assume only $L$ different values the computational effort can be tolerated. However, eq.(15) will still not lead to any meaningful result if one uses a conventional MC Method with single spin update. The reason are the cancellations in the sum involved in eq.(15) necessary to obtain a finite value for $g_{R, L}$. The corresponding fluctuations in each single measurement prevent any convergence of the average value inside present computing facilities. These fluctuations can be avoided by using a cluster algorithm. Its merit is not so much the reduced autocorrelation time between different configurations because this is balanced by the better vectorization of the local algorithms [11], but the existence of improved estimators for spin expectation values as (13). For the single cluster method the estimator for the correlation function can be written as (see Appendix B)

$$
g_{R, L}\left(k_{0}\right)=\left\{\frac{1}{s}\left|\sum_{x \in C} e^{-i\left(x \cdot k_{R, L}\right)}\right|^{2}\right\} .
$$

\{\} means an average over all single clusters $C$ and $s$ denotes the number of sites in $C$. Comparing eq.(15) and eq.(16) we notice the absence of the spin factor $\sigma_{x}$ which is due to the fact that all cluster spins have the same value. Moreover the sum over $x$ extends only over the cluster instead of the whole lattice as in eq.(15). Since the size of a cluster in the high temperature phase is governed by the correlation length $\xi$, the phase factor will not vary substantialy for $k_{0} \xi$ of $O(1)$ which is precisely the region we are interested in an application of eq.(16). Since the cancellations are only needed to render $g_{R, L}$ to be an decreasing function of $k_{0}$ they are no problem at all. Note that this improvements hold only for cases where the magnetization is small. For $T<T_{c}$ and finite $m$ a fraction $O(m)$ of the clusters will extend over the whole lattice and the same problems as in the single spin MC will reappear. Therefore we can use our method in the ordered phase in a narrow region of $T_{c}-T$ only.

For an error estimate one has to keep in mind that the autocorrelation time $t_{a}$ for a cluster algorithm even much smaller than for single spin update may not be small absolutely. Therefore we divide the number of generated clusters into $n_{B}$ blocks of size $n_{s}$. In each block the average of any observable is taken. The final mean value of the observable and its error are calculated 
from the different means of the blocks. This procedure will protect against autocorrelation times $t_{a}<n_{s}$. Typically we take a ratio $n_{s} / n_{B} \sim 100-500$. Also functions of observables (f.e. $g_{L}^{-1}$ ) including their error may be easily estimated.

\section{Results from numerical simulations}

In $d=2$ exact expressions for the behaviour of $\hat{g}^{-1}(k)$ at small values of $\mathrm{k}$ are known. For a comparism we used the cluster algorithm on a $160 \times 160$ lattice to simulate $1 / g_{R, L}$. Typically we generated $10^{5}$ clusters in the high temperature phase and $10^{4}$ clusters in the ordered phase. The range of temperatures $\tau= \pm 0.1, \tau= \pm 0.08, \tau= \pm 0.05$ and $\tau= \pm 0.03$ was motivated because the correlation length must be small compared to the lattice size in order to avoid any finite size corrections. In Fig. 1 we show $g_{R}^{-1}(k) \tau^{-7 / 4}$ as function of $\sin ^{2}(k / 2)$ for various temperatures $T>T_{c}$. Superimposed are straight line fits to points restricted to $\xi^{2} k^{2}<1$. For $\tau \geq 0.08$ the data exhibit a linear behaviour in $\sin ^{2} k / 2$ in the whole $k$ region. Approaching $T_{c}$ deviations from this behaviour become important in agreement with the high temperature expansion. $g_{L}^{-1}(k)$ exhibits the same behaviour. Since the statistical accuracy of $g_{R}$ is much better and the extrapolation of $g_{L}$ to $k \rightarrow 0$ is compatible with the one obtained from $g_{R}$ we discuss only the latter. The straight line fits yield us values for $\chi$ and $\xi^{2}$ according eq.(4). These fits are represented by the lines in Fig. 1. The resulting $\chi$ and $\xi$ are shown in Fig. 2 on a double $\log$ scale as function of $\tau$. The lines give the exact theoretical result [15, 16, 17], which agree well with the extrapolations of our data. The same analysis can be done for $T<T_{c}$. Due to the non vanishing magnetization less statistic is available and the quality is poorer as compared to $T>T_{c}$. The deviation from a straight line behaviour sets in much earlier than for $T>T_{c}$, as can be seen from the big difference of data and the corresponding lines fitted to the small $k$ region. From the fits we obtain $\chi$ and $\xi$ which are also shown in Fig.2. Again we notice the agreement with the exact results [15, 16, 17]. The value of $g_{R}^{-1}(0)$ can be translated via eq.(11) in a value for the magnetization. The values for $m$ are divided by the theoretical value [13, 14]

$$
m_{t h}=\left(\frac{\sinh ^{4}(2 / T)-1}{\sinh ^{4}(2 / T)}\right)^{1 / 8} .
$$


As one sees from Fig. 2, this ratio is compatible with 1 inside the small errors. In this case straight line fits to $\mathrm{m}$ are not very meaningful, since eq. (17) predicts a substantical deviation from the power law (5) in our range of $\tau$ values. To demonstrate the statistical accuracy we fit our values of $\chi$ and $\xi$ with the power laws (5). In fit $\mathrm{A}$ we fix the exponents to the theoretical values $\gamma=7 / 4$ and $\nu=1$, in fit $\mathrm{C}$ we fit in addition the exponents $\gamma$ resp. $\nu$ simultaneously to the data for $T \gtrless T_{c}$. The resulting parameters are displayed in table 1 together with the known exact values, resp. the values from the series expansion for the effective correlation length $f_{ \pm}$from ref [2, 3]. Both agree within the statistics which shows that the cluster algorithm leads to reasonable values for the correlation function.

In $d=2$ we can test apart from the behaviour near $T_{c}$ also the results of the high temperature expansion. If the coefficients $H_{n}$ for $n>2$ in the expansion of $\hat{g}^{-1}$ can be neglected , $a_{0}$ and $a_{1}$ can be determined from $g_{L}$ and $g_{R}$. The experimental value of coefficients $a_{0}$ and $a_{1}$ are obtained by eq. (12). These values divided by the series expansions (24) are shown in Fig. 4 as function of $1 / T$ for various values of the lattice size $L$. Below $1 / T=0.40$ the first two coefficients in the expansion (11) computed by the high temperature series give a good description of the correlation function which can be used in practical applications [18]. The deviations above 0.4 are not due to finite size effects. They signal not so much a breakdown of the series expansion for $a_{0,1}$ but rather the appearance of higher coefficients $a_{n}$ which spoil the relations (12) between $g^{-1}$ and $a_{0,1}$. This is corroborated by the values of $a_{2}$ obtained by eq.(12) which are zero below $1 / T=0.40$ and quickly exceed the value expected from eq.(24) by an order of magnitude. Whereas finite size corrections are negligiable for leading effects discussed up to now, a test of the Fisher hypothesis $\hat{g}(k)$ being a function of the lattice generating function only is very sensitive on small finite size corrections. This is because any deviation from the relation (12) can be a small effect only and the cancellations necessary to suppress the low order $1 / T$ terms in $H_{n}$ are no longer effective at order $1 / L$. We can control these corrections by the number of clusters with loop number [20] non zero (clusters wrapping around the lattice at least once). Requiring the fraction of those clusters to be less than $10^{-6}$ we have to choose on a $160 \times 160$ lattice an inverse temperature not larger than 0.42 . In order to make a small effect visible, we 
divide both $g_{L}^{-1}\left(\sin ^{2} \frac{k_{0}}{2}\right)$ and $g_{R}^{-1}\left(\frac{1}{2} \sin ^{2} \frac{k_{0}^{\prime}}{2}\right)$ by a common linear factor $\mathrm{R}$

$$
R=0.005+2.63 \sin ^{2} k_{0} / 2
$$

obtained by a fit to $g_{L}^{-1}$. In Fig. $5 g_{L}^{-1}\left(\sin ^{2} \frac{k_{0}}{2}\right) / R$ and $g_{R}^{-1}\left(\frac{1}{2} \sin ^{2} \frac{k_{0}^{\prime}}{2}\right) / R$ are shown as function of $\sin ^{2} \frac{k_{0}}{2}$. If $g^{-1}$ would be linear in $\sin ^{2} \frac{k_{0}}{2}$, both data points would fall on a constant line at 1 . The deviation from 1 signalizes the appearance of higher coefficients $H_{n}$ with $n \geq 2$ as expected from the series expansion. If the hypothesis (12) is true, both sets of data points have to agree, which is obviously not the case inside our errors. Since at the level of accuracy of $10^{-3}$ finite size corrections can be neglected, our data are in disagreement with Fishers conjecture.

For $d=3$ we restrict ourselves to the behaviour near $T_{c}=1 / 0.22165$ [21, 22]. The computational effort increases rapidly with $L$, especially in the ordered phase at $T<T c$. The data for a $40^{3}$ lattice shown in Fig. 6 needed $\sim 30 \mathrm{~h}$ on a CRAY YMP. Since the correlation lengths are smaller in $d=3$, we can cover the same range of $\tau$ as in $d=2$. As before the function $g_{R}\left(k_{0}\right)=\hat{g}\left(k_{0}, k_{0}, k_{0}\right)$ has smaller errors than $g_{L}$. We do not show a plot of $g_{R}$, since its behaviour is very similar to the $d=2$ case. Performing linear fits in the small $k$ region we obtain $\chi(\tau), \xi(\tau)$ and $m(\tau)$ shown in Fig. 6 on a double log scale. From the linear behaviour we see that the power laws predicted by eq.(5) are well satisfied. To obtain values for exponents and amplitudes we performed the following fits with or without correction to scaling:

$$
\begin{aligned}
\chi_{ \pm} & =C_{ \pm}|\tau|^{-\gamma}\left(1+a_{ \pm}|\tau|\right) \\
\xi_{ \pm} & =f_{ \pm}|\tau|^{-\nu}\left(1+a_{ \pm}^{\prime}|\tau|\right) \\
m & =B(-\tau)^{\beta}\left(1+b_{ \pm}|\tau|\right) .
\end{aligned}
$$

In principle the correction terms should be parametrized by nonlinear power laws, but within our statistics a determination of an exponent in the nonleading term is not possible. We checked that replacing $|\tau|$ by $|\tau|^{1 / 2}$ in the correction does not change the values of exponents or amplitudes. In fit A we fix the exponents to the generally accepted values [21, 22, 12] and leave out the corrections which are allowed in fit B. The fits $\mathrm{C}$ and $\mathrm{D}$ are similar to $\mathrm{A}$ and $\mathrm{B}$ except that also the exponents $\gamma, \nu$ and $\beta$ are free parameters. The resulting values and the corresponding $\chi^{2} / D o F$ are given in table II. In the fits to $\chi$ and $m$ correction terms are definiteley needed, whereas for $\xi$ 
inclusion of the latter does not improve $\chi^{2}$. In the case of the susceptibility variation of $\gamma$ does not reduce $\chi^{2} / D o F$. Therefore we believe that fit $B$ is the most reliable one and is also shown in Fig. 6. The values for the amplitudes are in agreement with the series expansion (last column in table II). Variation of the exponent $\nu$ for the correlation length leads to a value very close to the accepted value of $\nu$. Since including corrections in fit B did neither change the values of $f_{ \pm}$nor improve $\chi^{2} / D o F$ fit $\mathrm{D}$ should not be trusted so much. In this case we adopt fit $\mathrm{C}$ as the most reliable one. A common feature of fit $\mathrm{A}, \mathrm{B}$ and $\mathrm{C}$ is a consistent $5 \%$ deviation in $f_{-}$from the series expansion. The universal ratio (from fit C) $f_{+} / f_{-}$

$$
f_{+} / f_{-}=2.063(12)
$$

disagrees with ref [12, 7], but is in agreement with a recent calculation using renormalized perturbation expansion in $d=3$ [5] and with experimental data [6]. In the case of $m$ fit $\mathrm{D}$ is definitely preferred due to the acchieved $\chi^{2} / D o F$. Both exponent $\beta$ and the amplitude $B$ are somewhat smaller as compared to the series expansion but are consistent within the errors. In table II our final values for the amplitudes are underlined. With the exception of $f_{-}$ the cluster Monte Carlo method yielded values compatible with the series expansion with similar accuaracy.

\section{Conclusion}

Due to fluctuations the correlation function $g$ in Ising models for $d>1$ cannot be determined with local Monte Carlo methods except for rather modest lattice sizes. Since the improved estimators in a cluster algorithm exhibit much less fluctuation the fourier transform of $g$ can be measured. We used the known results in $d=2$ to demonstrate the validity of our method. As a byproduct we show that a hypothesis of Fisher may not be true. In $d=3$ our

simulations agree with the values expected from series expansion except for the amplitude $f_{-}$of the effective correlation length in the ordered phase. The universal ratio $f_{+} / f_{-}=2.06(1)$ is in agreement with recent field theoretical estimates.

\section{Acknowledgements:}


One of us (P.Z.) thank the University of Kiel for a grant. Computing time on a CRAY YMP was provided by HLRZ at Jülich.

\section{A High temperature expansion}

Taking the fourier transform of eq.(11) and comparing the coefficients of $\cos \left(k_{1} n\right), \cos \left(k_{2} m\right)$ with eq.(10) one finds

$$
\begin{aligned}
& a_{0}=1-H_{0}(T)-4 H_{2}(T) \\
& a_{1}=H_{1}(T) \\
& a_{2}=H_{2}(T) .
\end{aligned}
$$

$H_{i}$ have been given in [2] in terms of powers of $v=\operatorname{tgh}(1 / T)$ which leads to

$$
\begin{aligned}
& a_{0}=1+4 v^{2}+12 v^{4}+44 v^{6}+188 v^{8}+836 v^{10}+o\left(v^{12}\right) \\
& a_{1}=v\left(1+v^{2}+5 v^{4}+21 v^{6}+96 v^{8}+401 v^{10}\right)+o\left(v^{13}\right) \\
& a_{2}=4 v^{10}+o\left(v^{12}\right)
\end{aligned}
$$

\section{B The improved estimator}

The fourier transform of eq.(14) is

$$
\hat{g}(k)=\frac{1}{L^{d}} \sum_{x, y} e^{i k(x-y)}\left\langle\sigma_{x} \sigma_{y}\right\rangle
$$

For a Swendsen Wang [9] cluster decomposition of the lattice we have

$$
\left\langle\sigma_{x} \sigma_{y}\right\rangle=\sum_{C, C^{\prime}} \Delta_{x}(C) \Delta_{y}\left(C^{\prime}\right)\left\langle\sigma_{C} \sigma_{C^{\prime}}\right\rangle
$$

$\Delta_{x}(C)$ takes the value 1 if $x \in C$ and 0 otherwise. Noting that cluster spins are independent variables

$$
=\sum_{C} \Delta_{x}(C) \Delta_{y}(C)
$$


SO

$$
\hat{g}(k)=\frac{1}{L^{d}}\left\{\sum_{C}\left|\sum_{x \in C} e^{i k x}\right|^{2}\right\}_{S W} .
$$

The $1 / L^{d} \sum_{C}$ in the Swendsen Wang algorithm translates into $1 / s$ for a single cluster algorithm [23] so

$$
\hat{g}(k)=\left\{\frac{1}{s}\left|\sum_{x \in C} e^{i k \cdot x}\right|^{2}\right\}
$$

from which we obtain eq.(16). 
table I

\begin{tabular}{|l|c|c|c|}
\hline & $\mathrm{A}$ & $\mathrm{C}$ & \\
\hline \hline$\gamma$ & $7 / 4$ & $1.753(4)$ & $7 / 4$ \\
\hline$C_{+}$ & $0.98(2)$ & $0.97(9)$ & 0.962582 \\
\hline$C_{-}$ & $0.0247(3)$ & $0.025(3)$ & 0.025537 \\
\hline \hline$\nu$ & 1 & $0.99(3)$ & 1 \\
\hline$f_{+}$ & $0.558(4)$ & $0.55(2)$ & $0.56702(5)$ \\
\hline$f_{-}$ & $0.16(3)$ & $0.18(2)$ & $0.175(5)$ \\
\hline \hline
\end{tabular}

table II

\begin{tabular}{|l|c|c|c|c|c|}
\hline & $\mathrm{A}$ & $\mathrm{B}$ & $\mathrm{C}$ & $\mathrm{D}$ & series [12] \\
\hline \hline$\gamma$ & 1.237 & $\underline{1.237}$ & $1.168(10)$ & $1.235(44)$ & 1.237 \\
\hline$C_{+}$ & $1.198(5)$ & $1.093(13)$ & $1.432(33)$ & $1.12(19)$ & $1.103(1)$ \\
\hline$C_{-}$ & $0.191(4)$ & $0.211(11)$ & $0.237(11)$ & $0.219(41)$ & $0.223(3)$ \\
\hline$\chi^{2} / D o F$ & $81 / 18$ & $18 / 16$ & $29 / 17$ & $18 / 15$ & - \\
\hline \hline$\nu$ & 0.629 & 0.629 & $0.628(1)$ & $0.618(6)$ & 0.629 \\
\hline$f_{+}$ & $0.4997(2)$ & $0.4995(8)$ & $0.501(2)$ & $0.522(11)$ & $0.496(4)$ \\
\hline$f_{-}$ & $0.2415(8)$ & $0.238(2)$ & $0.243(1)$ & $0.251(8)$ & $0.251(1)$ \\
\hline$\chi^{2} / D o F$ & $64 / 16$ & $62 / 14$ & $63 / 15$ & $59 / 13$ & - \\
\hline \hline$\beta$ & 0.330 & 0.330 & $0.301(2)$ & $0.319(5)$ & 0.330 \\
\hline$B$ & $1.632(2)$ & $1.686(4)$ & $1.478(9)$ & $1.608(37)$ & $1.71(2)$ \\
\hline$\chi^{2} / D o F$ & $263 / 11$ & $35 / 9$ & $35 / 10$ & $24 / 8$ & - \\
\hline
\end{tabular}




\section{Figure captions}

Fig. 1: The inverse fourier transform of the correlation function $g_{L}^{-1}(k)$ above $T_{c}$ as function of $\sin ^{2} \frac{k}{2}$ for $\tau=0.1,0.08,0.05$ and 0.03 (from bottom to top). The straight lines are fits to the data for $\xi^{2} k^{2}<1$.

Fig. 2: $d=2$ : The susceptibility $\chi$, the effective correlation length $\xi$ and the magnetization $m$ divided by eq.(17) as function of $|\tau|$ on a double log scale. \pm indicates the sign of $T-T_{c}$. Solid lines represent the fit $\mathrm{C}$ (fit A) to $\xi(\chi)$ described in the text. The dotted line is the constant 1 .

Fig. 3: The inverse fourier transform of the correlation function $g_{L}^{-1}(k)$ below $T_{c}$ as function of $\sin ^{2} \frac{k}{2}$ for $\tau=-0.1,-0.08,-0.05$ and -0.03 (from bottom to top). The straight lines are fits to the first two $k$ values with $k \neq 0$.

Fig. 4: The coefficients $a_{0}$ and $a_{1}$ from eq.(12) divided by the series expansion (24) for different values of the lattice size $L$ as function of $1 / T$. The critical temperature is indicated by the arrow.

Fig. 5: The linear $g_{L}\left(k_{0}\right)$ and the radial $g_{R}\left(k_{0}^{\prime}\right)$ correlation function at $1 / T=0.42$ as function of $\sin ^{2} \frac{k_{0}}{2}$. If Fishers' hypothesis holds both have to be equal.

Fig. 6: $d=3$ : The susceptibility $\chi$, the effective correlation length $\xi$ and the magnetisation $m$ as function of $|\tau|$ on a double log scale. \pm indicate the sign of $T-T_{c}$. The straight lines correspond to fit B (fit C, fit D) for $\chi(\xi, m)$. 


\section{References}

[1] B.M. McCoy and T.T. Wu The two dimensional Ising model, Harvard University Press, Cambridge, Massachusetts 1973

[2] M.E. Fisher and R.J. Burford, Phys. Rev. 156 (1967),583

[3] H.B. Tarko and M.E. Fisher Phys. Rev. B 11 no.3 (1975),1217

[4] C. Domb and M.S. Green 'Phase Transitions and critical Phenomena" Vol. 3 Academic Press

[5] G. Münster and J. Heitger MS-TPI-94-01, hep-lat 9402017 (1994)

[6] V. Privman, P.C. Hohenberg and A. Aharony in 'Phase Transitions and critical Phenomena' eds. C. Domb and J.L. Lebowitz Academic Press

[7] E. Brezin,J.C. Le Guillou, J. Zinn-Justin’ Phys. Lett. 36,(1976),1351

[8] N. Metropolis, A.W. Rosenbluth, M.N. Rosenbluth, A.H. Teller and E. Teller J. Chem. Phys. 9, (1953),706

[9] R.H. Swendsen and J.S. Wang, Phys. Lett. 58,(1987),86

[10] U. Wolff Phys. Rev. Lett. 62,(1989),361

[11] N. Ito and G.A. Kohring Int. Journal of Modern Physics C

[12] A.L. Liu and M.E. Fisher, Physica A 156, (1989),35

[13] C.N. Yang Phys. Rev. 85,(1952),808

[14] L. Onsager in "Critical Phenomena in Alloys, Magnets and Superconductors" McGraw-Hill New York (R.E. Mills, E. Ascher and R.I.Jaffe eds.)

[15] E. Barouch, B.M. McCoy and T.T. Wu Phys. Rev. Lett. 31, (1973),1409

[16] D.B. Abraham Phys. Lett. 43 A,(1973),163

[17] R.J. Baxter "Exactly solved Models in Statistical Mechanics" Academic Press London 
[18] M. Schlüter and F. Wagner Phys. Rev. E 49 no.2 (1994)

[19] f.e. G. Bhanot, D. Duke and R. Salvador, J. Stat. Phys. 44, (1986),985

[20] C. Ruge, S. Dunkelmann and F. Wagner Phys. Rev. Lett. 69, (1992),2465

[21] A.M. Ferrenberg and D.P. Landau Phys. Rev. B 44, no.10, (1991),5081

[22] C.F. Baillie, R. Gupta, K.A. Hawick and C.S. Pawley, Phys. Rev. B 45, (1992), 10438

[23] C. Ruge, S. Dunkelmann, J. Wulf and F. Wagner, J. Stat. Phys. $\mathbf{7 3},(1993), 293$ 
This figure "fig1-1.png" is available in "png" format from: http://arXiv.org/ps/hep-lat/9403009v1 


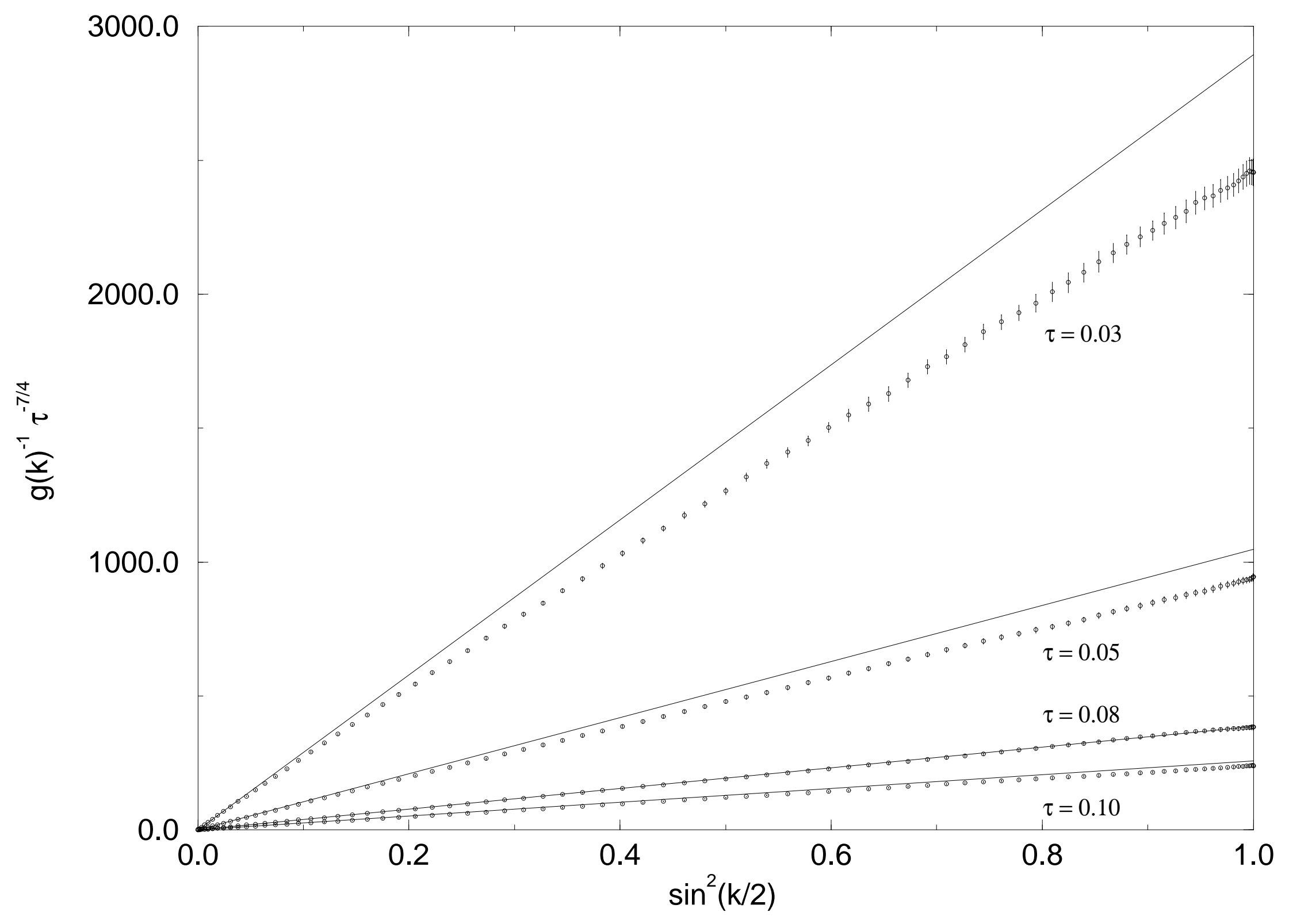


This figure "fig1-2.png" is available in "png" format from: http://arXiv.org/ps/hep-lat/9403009v1 


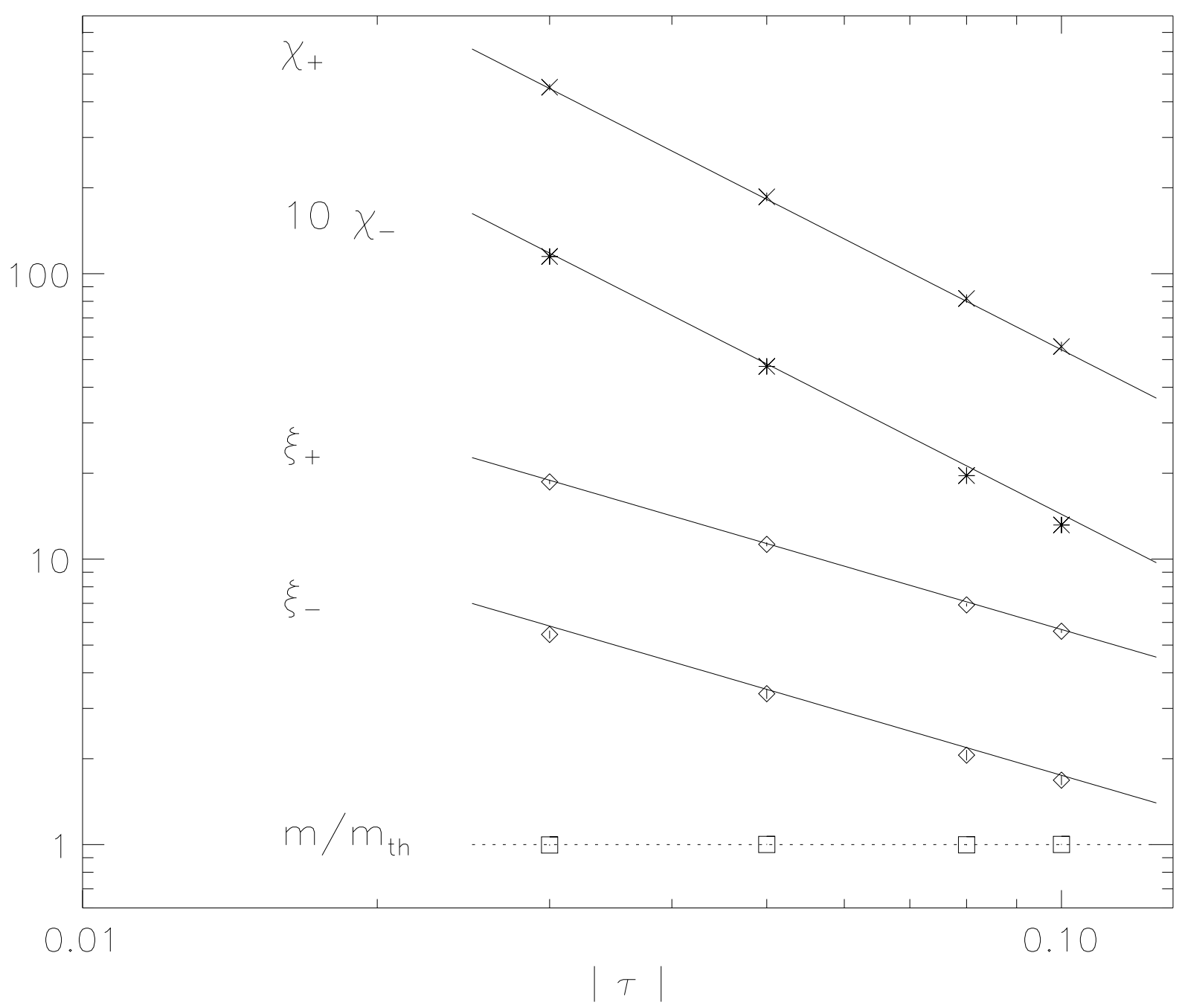


This figure "fig1-3.png" is available in "png" format from: http://arXiv.org/ps/hep-lat/9403009v1 


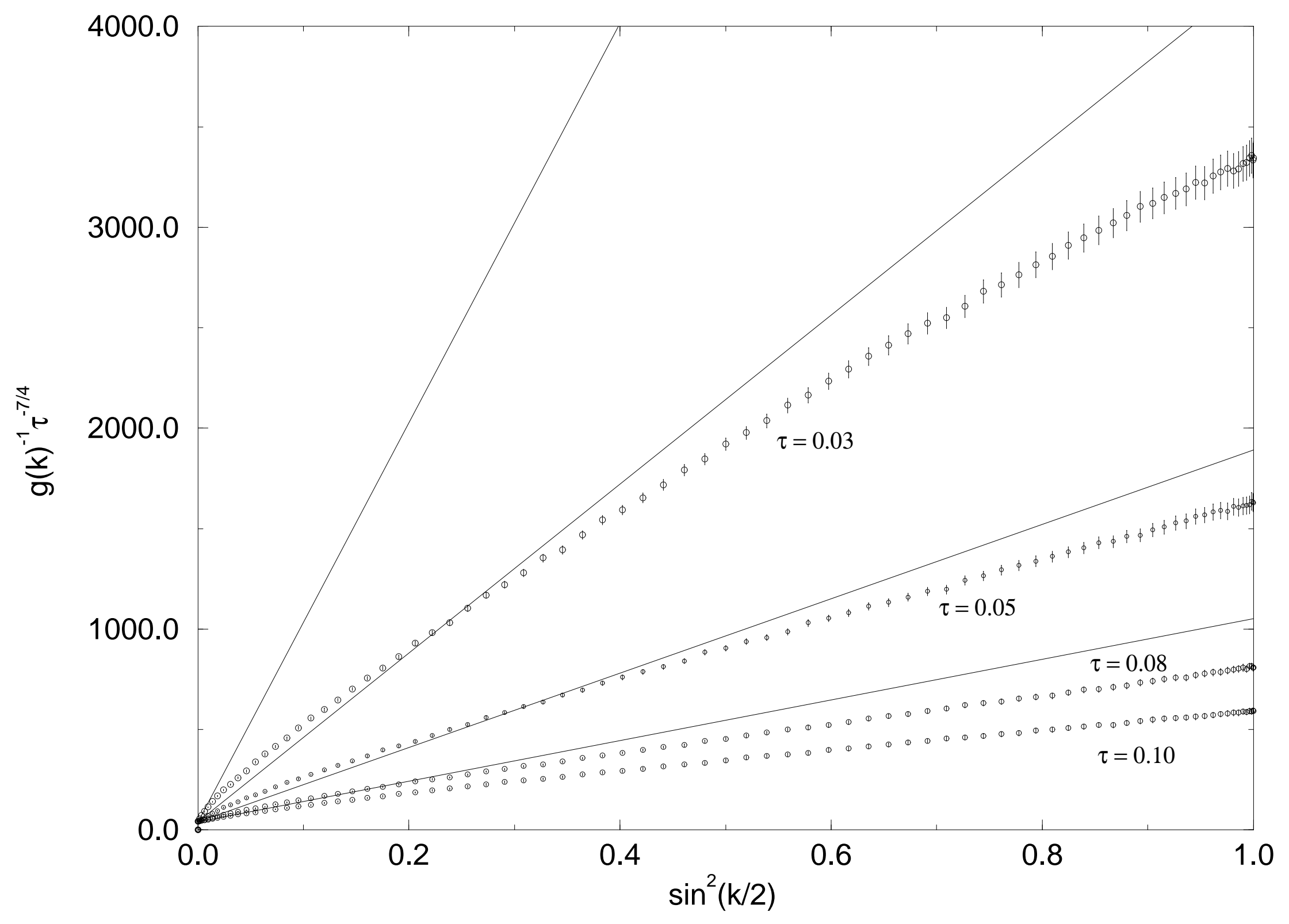


This figure "fig1-4.png" is available in "png" format from: http://arXiv.org/ps/hep-lat/9403009v1 


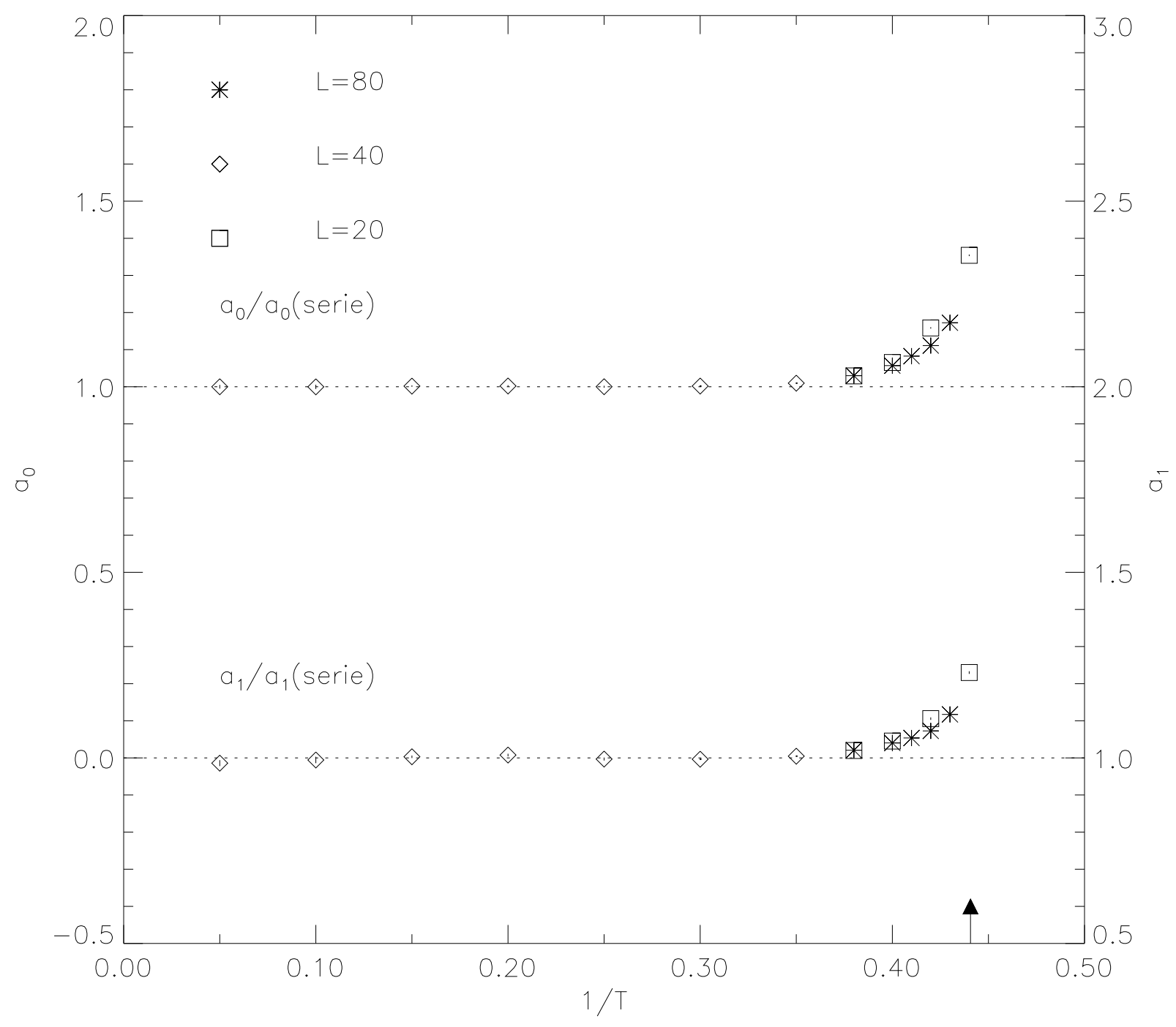


This figure "fig1-5.png" is available in "png" format from: http://arXiv.org/ps/hep-lat/9403009v1 


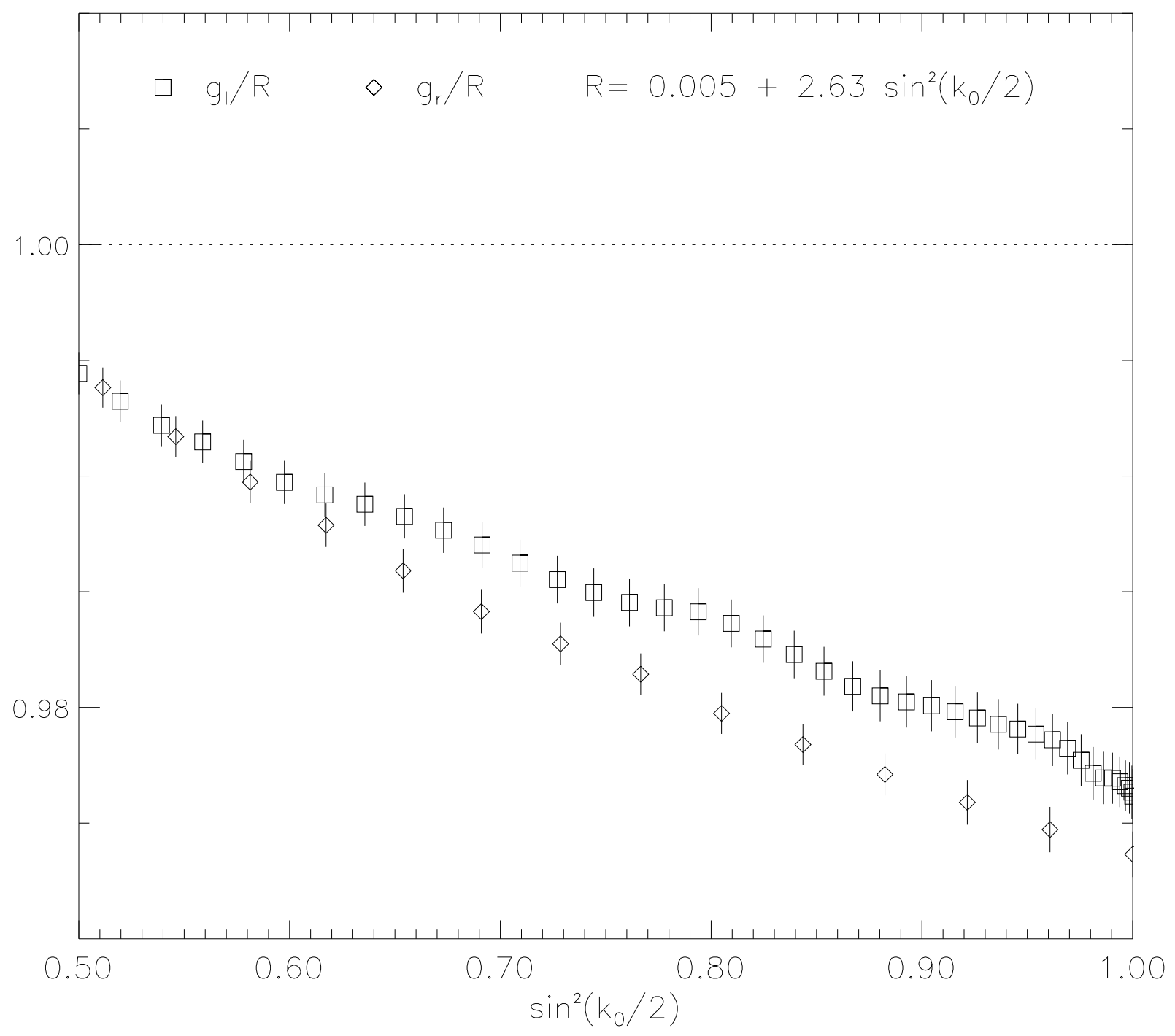


This figure "fig1-6.png" is available in "png" format from: http://arXiv.org/ps/hep-lat/9403009v1 


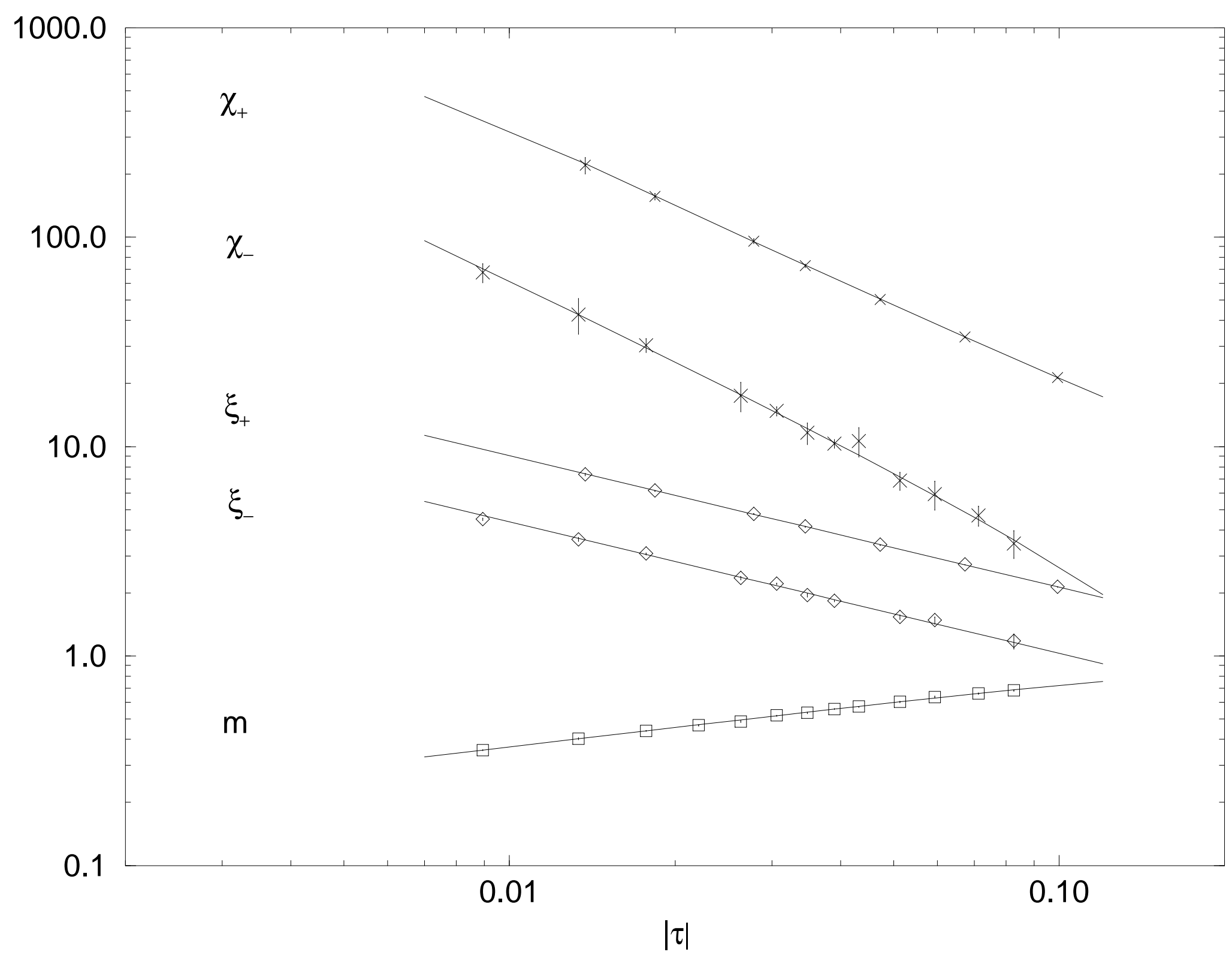

